\title{
Precautions against B Virus Infection
}

\author{
F. T. PERKINS,* PH.D. ; E. G. HARTLEY,* M.R.C.V.S.
}

Brit. med. F., 1966, 1, 899-901

$\mathrm{B}$ virus infection in monkeys is a benign disease causing minor or superficial lesions of a herpes-like character in the mouth or on the tongue (Fig. 1). Most of the monkeys imported into this country for laboratory purposes appear to be free from $B$ virus infection, but occasionally a batch may be received in which a high proportion are infected (Hartley, 1964a). It is

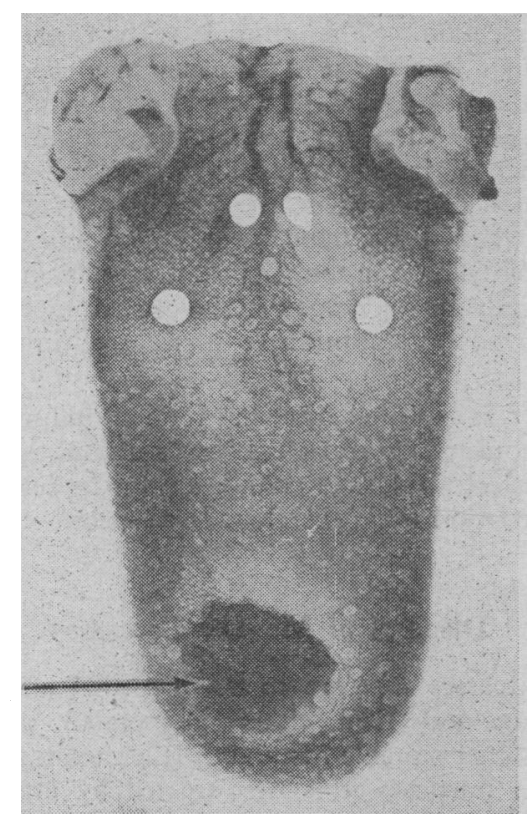

FIG. 1.-B virus lesion on the tongue of a monkey.

generally believed that monkeys without lesions are not infected, although there are no data to prove this. In man, B virus infection is extremely rare, but when it occurs it can be fatal. Over the last 35 years at least 15 cases have been reported, and 13 of these were fatal. It is thought that the disease was contracted from infected monkeys, since all of the cases occurred in people who had been in contact with monkeys or who had been working with monkey tissue, and it was known that seven of them had either been bitten or scratched.

The risk of man receiving a fatal infection of $B$ virus is thus very slight, but nevertheless it is important that to protect the personnel from infection adequate precautions are taken in laboratories where monkeys are used. We felt it would be useful to those who are not familiar with the handling of monkeys to outline the procedures adopted in our laboratory for dealing with this problem.

\section{Importation of Monkeys}

Although $B$ virus lesions have been seen only in monkeys received from India, Africa, and the Far East, all monkeys are regarded as being potentially infectious, since contact between non-infected and infected species may occur in transit. If it

* Division of Immunological Products Control, Medical Research Council Laboratories, Hampstead, London. is not important which species of monkeys is used, then New World monkeys which have never been in contact with Old World species are chosen. Under no circumstances are monkeys of different species mixed.

Since the disease is endemic in the jungles efforts are made to ensure that monkeys are inspected for lesions before they are dispatched. While the most careful plans can be made to implement this, it is not relied upon, and a further thorough inspection is made when the monkeys are received. Such an inspection is made on anaesthetized monkeys by a qualified person familiar with $\mathrm{B}$ virus infection, and all monkeys with specific lesions are destroyed immediately.

\section{Sedation of Monkeys}

Satisfactory sedation of monkeys is achieved by an intramuscular injection of phencyclidine hydrochloride $(2 \mathrm{mg} . / \mathrm{kg}$. body-weight). The effect of this dose is to reduce response to environment, reduce aggressiveness, and produce recumbent immobilization. This enables a monkey to be handled for many minor procedures, such as oral examination or withdrawal of blood. However, some of the simple reflexes, such as corneal and patellar, are not completely eliminated and the eye may remain open. Care is thus taken when first approaching a monkey after inoculation to make sure that sedation has taken place, which is usually in about five to ten minutes and lasts for one to two hours.

The drug can also be administered orally at a dose rate of up to $3 \mathrm{mg}$. $/ \mathrm{kg}$., but its sedative effects are slow in developing and may take 30 to 45 minutes or longer. Overdosage with phencyclidine on a massive scale can cause death, but modest overdosage can only produce convulsions, which do not appear to be lethal, particularly if the animal has received oxygen. There is no antidote.

The group of drugs referred to as "tranquillizers" are less reliable in action and do not necessarily possess sedative qualities ; indeed, some animals become hyperexcitable under the influence of these drugs when stimulated. With acceptance of this caution, tranquillizers which have been used for sedation are promazine, $4-6 \mathrm{mg} . / \mathrm{kg}$., given by the intramuscular route, and chlorpromazine, $1.5 \mathrm{mg} . / \mathrm{kg}$., given by either the intravenous or the intramuscular route. Both these drugs can be obtained in a suitable form for oral administration. Oral or parenteral barbiturates in the appropriate dose also have a sedative effect, but they are not so readily available.

\section{Housing of Monkeys}

On arrival the monkeys are housed in cages, no more than two per cage. The cages are kept in good condition and have no corroding areas that are difficult to clean. On no account are monkeys from different intake batches mixed in the same animal-room, even though they are of the same species. Before being used for experimental purposes the monkeys are kept in quarantine for a minimum of six weeks if caged singly, or eight weeks if housed two to a cage. The type of cage used is shown in Fig. 2 ; it was designed in this laboratory, and a blueprint of the design is available for those who may require it. The 
animal-house is kept clean and the excrement removed from the cages is incinerated. As each cage becomes empty it is disinfected, by either chemical or steam sterilization. As each room becomes empty it is disinfected by spraying all surfaces with an effective non-corrosive disinfectant before being used for a fresh intake of monkeys.

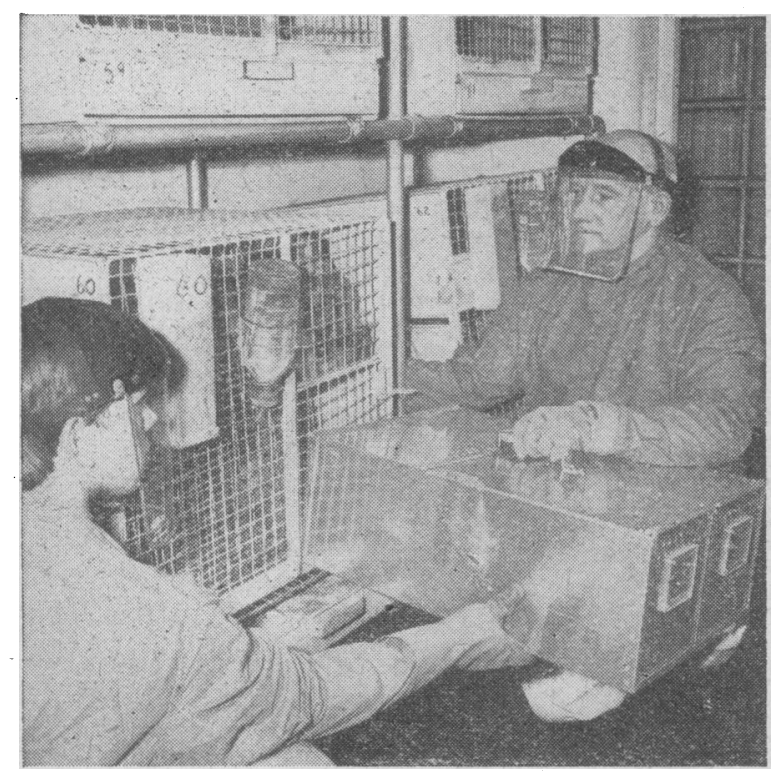

FIG. 2.-Manipulation of a monkey into a transport box.

\section{Protective Clothing}

All persons entering rooms in which monkeys are housed wear protective clothing to prevent any $B$ virus entering the body either through a scratch or abrasion, or as an aerosol. The same precautions are also taken by persons operating on monkeys or carrying out post-mortem operations. The clothing consists of a gown, disposable mask, goggles or visor, rubber boots, and disposable rubber gloves, preferably extending to the elbow. It is important that the gloves are not easily torn and equally important that they do not in any way restrict movement of the hand. All disposable clothing is discarded into a refuse-bin at the entrance of the animal-house, and gowns are placed in a receptacle, in which they are disinfected before being handled by the laundry.

Bowls of disinfectant, such as hypochlorite or surface-active agent, and footbaths of a phenolic or a surface-active disinfectant, are placed between animal-rooms for disinfection of gloved hands and boots before separate rooms are entered. Protein and faecal matter can inactivate many disinfectants, and it is important to ensure that a reliable disinfectant in an effective concentration is present throughout the working day.

Before leaving animal quarters all animal-handlers have a complete change of clothing and a shower.

\section{Handling of Monkeys}

In general, monkeys are handled only after they have been anaesthetized or suitably sedated. On no account does a person on his own attempt to handle a monkey; two persons are always present, one of whom is an experienced handler.

When monkeys are removed from their cages for experimental or other purposes a catch-box is used from which the monkey cannot escape. Fig. 2 shows the way in which a monkey is transferred from a cage to a catch-box. The design of a suitable container for the short-distance transfer of monkeys has been described by Hartley (1964b). It may be noted that the only opening in the cage is the bottom right quadrant, and in order to prevent the escape of the monkey the whole opening is covered by the flange of the catch-box.

A suitable method of giving an anaesthetic or sedative to a monkey by intraperitoneal or intramuscular injection without the necessity for handling the animal is to restrain the animal by use of a movable partition in the cage.

\section{Recapture of an Escaped Monkey}

The recapture of an escaped monkey is undertaken with the utmost caution, since the possibility of personnel being bitten is very great. If the animal has escaped from a cage every effort is made to limit further freedom by closing doors of rooms and corridors.

Recapture is best achieved by placing food as a bait in a trap cage. If this method takes too long and the animal is indoors, use is made of a ring net of stout cotton string tied at each intersection. The animal is best captured, when possible, at floor level by using the net in a scooping fashion rather than the downward "butterfly-catching" way. Once ensnared, the pole of the catching-net is given one or two twists to wrap the bag of the net around the pole and to seal off the mouth of the net to prevent the animal re-escaping. Alternatively, and when on the floor, the animal can be forced further into the net by using a broomstick horizontally in contact with the floor and advancing it against the captive monkey. In this position it is safe to administer a sedative injection to the animal.

If a monkey is captured by using food containing a sedative, the sedated monkey is approached with caution, as the action of orally administered sedatives cannot always be relied upon and the level of sedation must therefore be suspect.

\section{Disposal of Monkey Carcasses}

Monkey carcasses that are to be discarded are placed in a leak-proof bag and then either incinerated or completely destroyed by other suitable methods. If a carcass is wanted for demonstration purposes it is first treated by immersing it in $10 \%$ formalin, deep incisions in the muscle being made to facilitate penetration.

\section{Action to be Taken if Scratched or Bitten}

Any injury caused by a monkey or by glassware used for containing monkey-tissue receives immediate attention.

1. The wound is scrubbed clean with copious supplies of soap-and-water and made to bleed immediately.

2. The person reports to the accident-room, and the wound is cleaned with an antiseptic by the medical officer.

3. If a monkey has caused the injury the animal is identified, anaesthetized, and thoroughly examined for lesions in the mouth.

4. The general practitioner of the wounded person is warned about the injury and is asked to keep the patient under observation for three weeks (the incubation period for $B$ virus infection is about 20 days). In the event of the patient showing any signs of illness, such as those associated with influenza or muscular weakness, arrangements are made for him to be taken to an infectious-diseases hospital with an attached unit for treating respiratory paralysis. It has been reported by Breen et al. (1958) that cortisone therapy is important in cases of $B$ virus infection, and the attention of medical officers of hospitals may be drawn to this report. Appearance of vesicular lesions around the injury is a sign of $B$ virus infection, though these do not always occur. The disease has never been known to spread from man to man. 


\section{Summary}

The risk of fatal infections of B virus occurring in man as a result of the handling of monkeys or monkey tissue is very slight. It is important, however, in laboratories where monkeys are used to take precautions to protect the personnel from this infection. The precautions taken in our laboratory have been outlined and may be useful for those who are not familiar with the handling of monkeys.

We wish to thank the following for their advice in writing this account: Dr. A. J. Beale (Glaxo Laboratories), Dr. C. R. Coid (Royal College of Surgeons), Professor D. G. Evans (London School of Hygiene and Tropical Medicine), Mr. Oliver Graham-Jones
(Zoological Society of London), Dr. L. Goodwin (Nuffield Institute of Comparative Medicine), Mr. S. A. Keeble (Glaxo Laboratories), Dr. J. A. McFadzean (May and Baker Ltd.), Dr. Max McCowen (Eli Lilly Ltd), Dr. J. R. Napier (Royal Free Hospital), Mr. G. T. Tribe (Wellcome Research Laboratories), Dr. P. Stones (Pfizer Ltd.).

We especially wish to thank Mr. Keeble for the photograph of a B virus lesion and Mr. Oliver Graham-Jones for his advice on the sedation and recapture of an escaped monkey.

\section{REFERENCES}

Breen, G. E., Lamb, S. G., and Otaki, A. T. (1958). Brit. med. f., 2, 22.

Hartley, E. G. (1964a). Vet. Rec., 76, 555

Hartley, E. G. (1964a). Vet. Rec., 76, 555

\title{
Erythrocyte Sedimentation Rate in Elderly Hospital In-patients
}

\author{
R. V. BOYD,* M.B., M.R.C.P., M.R.C.P.ED. ; B. I. HOFFBRAND, $\dagger$ M.A., B.M., M.R.C.P.
}

It has been firmly established that the erythrocyte sedimentation rate (E.S.R.) in apparently healthy elderly subjects is higher than the usually accepted normal limits (Olbrich, 1948; Wilhelm and Tillisch, 1951 ; Renbourn, 1952 ; Vignon, 1958 ; Hilder and Gunz, 1964). This fact is not widely appreciated, however, in spite of the increasingly high proportion of elderly patients seen in clinical practice, and receives scant attention in standard textbooks of haematology. The cause of the " raised" E.S.R. is unknown and has been attributed to occult infections, especially urinary (Vignon, 1958 ; van Zonneveld, 1961 ), and to changes in the plasma proteins (Olbrich, 1948).

The present study was undertaken to determine the significance in the elderly of what would generally be accepted as an abnormally raised E.S.R.- that is, one above $20 \mathrm{~mm}$. in the first hour (Ansell and Bywaters, 1958). Our aims were twofold : (1) to see what contribution, if any, occult infections of the urinary tract make to the E.S.R. in the elderly, and (2) to determine the upper limit of normal of the E.S.R. performed as a routine laboratory test in elderly hospital in-patients.

\section{Methods}

The patients studied were those admitted to the geriatric wards of University College Hospital between January 1963 and March 1964. In addition to the routine history, physical examination, and pathological investigations indicated, all patients had the following tests: haemoglobin estimation; white-cell count and differential ; E.S.R. by a modification of the Westergren (1926) method, blood being drawn up to the $100 \mathrm{~mm}$. mark ; mid-stream specimen of urine with prior penile and vulval cleansing; chest $x$-ray examination. In randomly selected patients the Hyland latex fixation test for rheumatoid factor was performed.

The hospital notes of these patients were examined after discharge. A small number of patients were immediately excluded from further study: those under 65 years of age, those with inadequate history or physical examination recorded, those who did not have one or more of the investigations listed above, and those who were observed in the ward for less than 48 hours.

\footnotetext{
* Consultant Physician in Geriatrics, Greenwich District Hospital ; late Geriatric Registrar, University College Hospital, London.
} † Medical Registrar, University College Hospital, London.
We next excluded those patients with a known cause for an abnormal E.S.R. The diagnoses were usually the obvious ones, such as infections (excluding occult infections' of the urinary tract, see below), malignancy, recent visceral infarctions and venous thrombosis, cardiac failure, and rheumatoid arthritis. We rigorously excluded, as possible cases of chronic bronchitis, all patients with a history of chronic or recurrent cough and sputum, all who were seen to be coughing or producing sputum, and all with added sounds on auscultation of the lungs. Patients with a haemoglobin of less than $12.6 \mathrm{~g} . / 100 \mathrm{ml}$. or with pulmonary $x$-ray changes were also not included for further study.

There were 303 patients aged 65 and over who had full clinical and pathological investigations and who were observed in the ward for 48 hours or more.

Control groups of male and female patients under the age of 60 admitted to the general medical and neurological wards were studied. These patients had all the investigations listed above, and, judged by the same criteria, had no cause for an abnormal E.S.R.

Criteria of Infection of the Urinary Tract.-Any pyuria consisting of 6 or more cells per $1 / 6$ high-power field with or without growth, or any mid-stream specimen of urine yielding a pure growth without pyuria, was regarded as indicating an infection of the urinary tract.

\section{Results}

Of the 303 patients 38 ( 6 male, 32 female) had no apparent . cause for an abnormal E.S.R., and a further 33 (4 male, 29 female) no apparent cause apart from an occult infection of the urinary tract (in only 4 of these latter 33 patients was there a sterile pyuria, positive cultures being obtained in the remainder). The Table compares the findings in these two groups. This shows no significant difference either in the mean E.S.R. or in the numbers with a conventionally raised E.S.R. Thus occult infections of the urinary tract do not appear to make any significant contribution to the E.S.R. in the vast majority of elderly hospital in-patients.

Considering these two groups together, Fig. 1 shows that in the age group 65 and over there is no tendency for the high 